\title{
Immunotherapy transforms cancer treatment
}

\author{
Neeha Zaidi and Elizabeth M. Jaffee \\ Sidney Kimmel Comprehensive Cancer Center, Skip Viragh Center for Pancreas Cancer, The Bloomberg Kimmel Institute for Cancer Immunotherapy, Johns Hopkins University School of Medicine, \\ Baltimore, Maryland, USA.
}

ames Allison of the University of Texas MD Anderson Cancer Center and Tasuko Honjo of Kyoto University were jointly awarded the 2018 Nobel Prize in Physiology or Medicine for their pioneering discoveries that led to the development of immune checkpoint inhibitors, a class of drugs that have transformed the standard of care for many advanced malignancies. Immune checkpoint inhibitors are monoclonal antibodies that block the inhibitory action of $\mathrm{T}$ cell molecules, such as programmed death receptor-1 (PD-1) and cytotoxic $\mathrm{T}$ lymphocyte-associated protein 4 (CTLA-4), to allow T cells to recognize and destroy cancer cells. A number of these inhibitory monoclonal antibodies have gained approval by the FDA for the treatment of many different types of cancers, including melanoma, non-small cell lung cancer, renal cancer, lymphoma, and a subset of colon cancer, namely mismatch repair-deficient colon cancer. These checkpoint inhibitors have shown evidence of prolonged survival, and even cures, for some of these advanced cancers, which previously had very poor prognoses. These approvals have established the fifth pillar of cancer therapeutics, joining surgery, cytotoxic chemotherapy, molecularly targeted therapy, and radiation therapy.

For their biologic basis, these discoveries rely on the premise that $\mathrm{T}$ cell function is tightly regulated by both stimulatory and inhibitory checkpoints, a balance that strives to maintain self-tolerance and prevent autoimmunity. MHC molecules located on antigen-presenting cells, such as dendritic cells, present foreign tumor-specific antigens to $\mathrm{T}$ cells in the lymph nodes. $\mathrm{T}$ cell priming and activation occur when the MHC-peptide complex interacts with the $\mathrm{T}$ cell receptor (TCR), followed by the engagement of CD28 to B7.1 (CD80) or B7.2
(CD86) coreceptors. Effector T cells (Teffs) then traffic to and interact with tumor cells that present cognate antigens on MHC molecules, resulting in tumor cell lysis.

Allison studied CTLA-4, the first immune checkpoint molecule exploited for a therapeutic advantage. CTLA- 4 and its ligands B7.1 and B7.2 on antigen-presenting cells were originally discovered in the 1980s. CTLA-4 was found to be present on activated Teffs as well as on immunosuppressive Tregs (1). Early landmark studies showed that TCR stimulation is regulated by CD28 costimulatory signals, as well as inhibitory signals derived from CTLA-4. Notably, Allison found that CTLA-4 competed with $\mathrm{CD} 28$ for the $\mathrm{B} 7$ receptor and thereby prevented $\mathrm{CD}^{+}{ }^{+} \mathrm{T}$ cell activation (2). Given CTLA-4's function as a negative regulator of T cell activation, Allison's group came to the realization that blocking CTLA-4 with an antibody could release the brakes on $\mathrm{T}$ cell function. Many at the time were targeting CTLA- 4 for the treatment of autoimmune diseases, but in 1996, Allison's group published a seminal paper showing that an antagonist monoclonal antibody to CTLA-4 triggered antitumor immunity and resulted in the rejection of tumors in mice (3). Less than a decade later, the group reported the first human data documenting a robust antitumor response and clinical regression with an anti-CTLA-4 antibody in patients with advanced melanoma and ovarian cancer who had previously received a cancer vaccine (4). This study was followed by larger clinical trials that demonstrated clear overall survival benefits, culminating ultimately in 2011 in the approval of ipilimumab (Yervoy) for metastatic melanoma (5). The drug later gained FDA approval for adjuvant use in high-risk melanoma. As the initial immune checkpoint inhibitor to be approved, ipilimumab represents the first success story of

Conflict of interest: EMJ receives research funding from Bristol-Myers Squibb and Aduro Biotech, is on the advisory board for Genocea Biosciences, Adaptive Biotech, CSTONE, and DragonFly, and has the potential to receive royalties from Aduro Biotech.

Reference information: / Clin Invest. 2019;129(1):46-47. https://doi.org/10.1172/JCI126046.

the immune system being harnessed pharmacologically to a therapeutic advantage in cancer patients.

Meanwhile, in the early 1990s, while looking for genes that were involved in normal programmed cell death, Honjo and colleagues in Japan discovered a second immune checkpoint, PD-1 (6). Studies using subtractive hybridization showed that PD-1 was expressed only on immune cells and that disruption of the $P d 1$ gene in mice caused a lupus-like autoimmune syndrome. The findings collectively suggested that PD-1 was a negative regulator of immune responses (7). However, the precise mechanism for PD-1 regulation remained unknown until circa 2000, when its ligands, PD-L1 and PD-L2, were discovered and shown to be inhibitory to $\mathrm{T}$ cell activation $(8,9)$. In parallel studies, PD-L1 was found to be upregulated on tumor cells, suggesting that the interruption of PD-1 and its ligand could unleash an antitumor response. Honjo, along with other groups, provided the first lines of unequivocal evidence that the inhibition of PD- 1 or PDL- 1 using specific antibodies led to a robust antitumor response (10). These breakthrough observations would later underscore the development of two antibodies to PD-1, pembrolizumab (Keytruda) and nivolumab (Opdivo), for cancer immunotherapy. Both antibodies were FDA approved for advanced melanoma in 2014, and a number of positive clinical trials subsequently led to their approval for non-small cell lung cancer, small cell lung cancer, renal cell carcinoma, Hodgkin lymphoma, squamous cell carcinoma of the head and neck, urothelial carcinoma, mismatch repair-deficient colorectal cancer, and hepatocellular carcinoma. In addition, in 2015, atezolizumab, an anti-PD-L1 antibody, was approved for the treatment of non-small cell lung and urothelial cancers.

Allison's and Honjo's discoveries of checkpoint molecules and their demonstration of the antitumor immunity of targeted antibodies have not only profoundly impacted the care of cancer patients, but also spurred a renewed interest in the way 
we think about cancer evasion and response. Importantly, efforts are currently being made to understand why some patients have a durable response (20\%) while others ultimately relapse (80\%). Multiple mechanisms of resistance in those patients who initially respond have been elucidated and include both immune (e.g., loss of HLA molecules leading to reduced antigen presentation and $\mathrm{T}$ cell exhaustion) and genetic (e.g., changes in the neoantigen landscape) mechanisms. There is also an ongoing interest in combining multiple checkpoint inhibitors to increase response rates. Given their non-overlapping mechanisms of action, the co-blockade of PD-1 and CTLA-4 was found to result in increased 3-year overall survival rates (58\%) compared with, for example, PD-1 blockade alone $(52 \%)$, albeit with greater immunerelated adverse events (11). Of note, there are a number of other stimulatory and inhibitory checkpoints that are being targeted in combination with anti-PD-1 therapy to enhance clinical response.

Furthermore, there is a burgeoning interest in combining immune checkpoint inhibitors with other therapeutic agents, such as vaccines, chemotherapy, and radiotherapy, in cancers that are insensitive to checkpoint inhibitors at baseline. Notably, checkpoint inhibitors have been most successful in the treatment of patients with cancers such as melanoma and smokingassociated non-small cell lung cancer that are inherently rich in infiltrating $\mathrm{T}$ cells and harbor a high number of somatic mutations. A subset of these somatic mutations express neoantigens, which are tumor-specific proteins produced during cancer progression and are recognized as foreign by the immune system. However, the majority of aggressive cancers (>70\%), including pancreatic cancer and glioblastoma, do not respond at all to immune checkpoint therapy. These tumors typically have a low burden of mutations that express much fewer neoantigens and therefore fail to attract $\mathrm{T}$ cells into their immunosuppressive tumor microenvironment. Current studies are therefore focused on first using agents, such as vaccines, that may be capable of inducing $\mathrm{T}$ cells in the tumor and then adding in checkpoint inhibitors to optimize $\mathrm{T}$ cell function (12).

The idea of harnessing the immune system to fight cancer had been actively investigated since the early 1900 s when William Cooley attempted to cure advanced sarcoma with heat-inactivated bacterial toxins. However, until these seminal discoveries by Allison and Honjo, there had been no clinical benefit with immunotherapy. Indeed, checkpoint inhibitors have dramatically changed the way certain tumor types, notably those with a high mutational burden, are treated. In fact, there seems to be a direct correlation between tumor mutational burden and response to checkpoint inhibitor therapy (13). Current work is focused on how to maintain durable responses as well as to sensitize those tumor types that are immune insensitive at baseline to checkpoint inhibitor therapy. Toward this goal, combining checkpoint inhibitors with other therapeutic modalities, such as those aimed at immunosuppressive cells in the tumor microenvironment, have shown promise in preclinical and early clinical studies.

Address correspondence to: Elizabeth M. Jaffee, Bunting-Blaustein Cancer Research Building, Johns Hopkins University, 1650 Orleans Street, Room 4M07, Baltimore, Maryland 21231, USA. Phone: 410.955.2957; Email: ejaffee@jhmi.edu.
1. Brunet JF, et al. A new member of the immunoglobulin superfamily - CTLA-4. Nature. 1987;328(6127):267-270.

2. Krummel MF, Allison JP. CD28 and CTLA-4 have opposing effects on the response of T cells to stimulation. J Exp Med.1995;182(2):459-465.

3. Leach DR, Krummel MF, Allison JP. Enhancement of antitumor immunity by CTLA-4 blockade. Science. 1996;271(5256):1734-1736.

4. Hodi FS, et al. Biologic activity of cytotoxic T lymphocyte-associated antigen 4 antibody blockade in previously vaccinated metastatic melanoma and ovarian carcinoma patients. Proc Natl Acad Sci U S A. 2003;100(8):4712-4717.

5 . Hodi FS, et al. Improved survival with ipilimum$\mathrm{ab}$ in patients with metastatic melanoma. $\mathrm{NEngl}$ JMed. 2010;363(8):711-723.

6. Ishida Y, Agata Y, Shibahara K, Honjo T. Induced expression of PD-1, a novel member of the immunoglobulin gene superfamily, upon programmed cell death. EMBO J.1992;11(11):3887-3895.

7. Nishimura H, Nose M, Hiai H, Minato N, Honjo T. Development of lupus-like autoimmune diseases by disruption of the PD-1 gene encoding an ITIM motif-carrying immunoreceptor. Immunity. 1999;11(2):141-151.

8. Freeman GJ, et al. Engagement of the PD-1 immunoinhibitory receptor by a novel B7 family member leads to negative regulation of lymphocyte activation. J Exp Med. 2000;192(7):1027-1034.

9. Latchman Y, et al. PD-L2 is a second ligand for PD-1 and inhibits T cell activation. Nat Immunol. 2001;2(3):261-268.

10. Iwai Y, Ishida M, Tanaka Y, Okazaki T, Honjo T, Minato N. Involvement of PD-L1 on tumor cells in the escape from host immune system and tumor immunotherapy by PD-L1 blockade. Proc Natl Acad Sci U S A. 2002;99(19):12293-12297.

11. Wolchok JD, et al. Overall survival with combined Nivolumab and Ipilimumab in advanced melanoma. N Engl J Med. 2017;377(14):1345-1356.

12. Kinkead HL, et al. Combining STING-based neoantigen-targeted vaccine with checkpoint modulators enhances antitumor immunity in murine pancreatic cancer. JCI Insight. 2018;3(20):e122857.

13. Yarchoan M, Hopkins A, Jaffee EM. Tumor mutational burden and response rate to $\mathrm{PD}-1$ inhibition. N Engl JMed. 2017;377(25):2500-2501. 